\title{
Differential Equations Associated to the $S U(2)$ WZNW Model on Elliptic Curves
}

By

\author{
Takeshi SuzukI*
}

\begin{abstract}
We study the $S U$ (2) WZNW model over a family of elliptic curves. Starting from the formulation developed in [13], we derive a system of differential equations which contains the Knizhnik-Zamolodchikov-Bernard equations [1] [9]. Our system completely determines the $N$-point functions and is regarded as a natural elliptic analogue of the system obtained in [12] for the projective line. We also calculate the system for the 1-point functions explicitly. This gives a generalization of the results in $[7]$ for $\widehat{\mathfrak{s l}}(2, \mathbb{C})$-characters.
\end{abstract}

\section{§0. Introduction}

We consider the Wess-Zumino-Novikov-Witten (WZNW) model. A mathematical formulation of this model on general algebraic curves is given in [13], where the correlation functions are defined as flat sections of a certain vector bundle over the moduli space of curves. On the projective line $\mathbb{P}^{1}$, the correlation functions are realized more explicitly in [12] as functions which take their values in a certain finite-dimensional vector space, and characterized by the system of equations containing the Knizhnik-Zamolodchikov (KZ) equations [11]. One aim in the present paper is to have a parallel description on elliptic curves. Namely, we characterize the $N$-point functions as vector-valued functions by a system of differential equations containing an elliptic analogue of the $\mathrm{KZ}$ equations by Bernard [1]. Furthermore we write down this system explicitly in the 1-pointed case.

To explain more precisely, first let us review the formulation in [13] roughly. Let $\mathfrak{g}$ be a simple Lie algebra over $\mathbb{C}$ and $\hat{\mathfrak{g}}$ the corresponding affine Lie algebra. We fix a positive integer $\ell$ (called the level) and consider the integrable highest weight modules of $\hat{\mathfrak{g}}$ of level $\ell$. Such modules are parameterized by the set of highest weight $P_{\ell}$ and we denote by $\mathscr{H}_{\lambda}$ the left module corresponding to $\lambda \in P_{\ell}$. By $M_{g, N}$ we denote the moduli space of

Communicated by T. Miwa, February 9, 1995.

1991 Mathematical Subject Classification(s):

* Research Institute for Mathematical Sciences, Kyoto University, Kyoto, 606-01, Japan. 
$N$-pointed curves of genus $g$. For $\mathfrak{X} \in M_{g, N}$ and $\vec{\lambda}=\left(\lambda_{1}, \ldots, \lambda_{N}\right) \in\left(P_{\ell}\right)^{N}$, we associate the space of conformal blocks $\mathscr{V}_{g}^{\dagger}(\mathfrak{X} ; \vec{\lambda})$. The space $\mathscr{V}_{g}^{\dagger}(\mathfrak{X} ; \vec{\lambda})$ is the finite dimensional subspace of $\mathscr{H}_{\vec{\lambda}}^{\dagger}:=\operatorname{Hom}_{\mathbb{C}}\left(\mathscr{H}_{\lambda_{1}} \otimes \cdots \otimes \mathscr{H}_{\lambda_{N}}, \mathbb{C}\right)$ defined by "the gauge conditions". Consider the vector bundle $\tilde{\mathscr{V}}_{\boldsymbol{g}}^{\dagger}(\vec{\lambda})=\bigcup_{\mathfrak{x}_{\in \in \boldsymbol{M}, \boldsymbol{N}}}$ $\mathscr{V}_{g}^{\dagger}(\mathfrak{X} ; \vec{\lambda})$ over $M_{g, N}$. On this vector bundle, projectively flat connections are defined through the Kodaira-Spencer theory, and flat sections of $\tilde{\mathscr{V}}_{g}^{\dagger}(\vec{\lambda})$ with respect to these connections are called the $N$-point correlation functions (or $N$-point functions). In the rest of this paper we set $\mathfrak{g}=\mathfrak{s l}(2, \mathbb{C})=\mathbb{C} E \oplus \mathbb{C} F \oplus$ $\mathbb{C} H$ for simplicity, where $E, F$ and $H$ are the basis of $\mathfrak{g}$ satisfying

$$
[H, E]=2 E,[H, F]=-2 F,[E, F]=H .
$$

We identify $P_{\ell}$ with the set $\left\{0, \frac{1}{2}, \ldots, \frac{\ell}{2}\right\}$ by the mapping $\lambda \mapsto \frac{\lambda(H)}{2}$.

In the case of genus 0 , the space of conformal blocks is injectively mapped into $V_{\vec{\lambda}}^{\dagger}:=\operatorname{Hom}_{\mathbb{C}}\left(V_{\lambda_{1}} \otimes \cdots \otimes V_{\lambda_{N}}, \mathbb{C}\right)$ by the restriction map, where $V_{\lambda} \subset \mathscr{H}_{\lambda}$ denotes the finite dimensional irreducible highest weight left $\mathfrak{g}$-module with highest weight $\lambda$. This injectivity makes it possible to treat this model in a more explicit way as above, and the $N$-point functions are described by the vacuum expectation values of vertex operators.

On the other hand, in the case of genus 1 this injectivity does not hold, and in order to recover it we twist the space of conformal blocks by introducing a new parameter following [1][2][6][9]. Because of the twisting, any $N$-point function in genus 1 can be calculated from its restriction to $V_{\vec{\lambda}}$ (Proposition 3.3.2). It is natural to ask how the restrictions of the $N$-point functions are characterized as $V_{\vec{\lambda}}^{\dagger}$-valued functions. It turns out that the restricted $N$-point functions satisfy the equations (E1)-(E3) in Proposition 3.3.3. These equations are essentially derived by Bernard [1] for traces of vertex operators

$$
\operatorname{Tr}_{\mathscr{H}_{\mu}}\left(\varphi_{1}\left(z_{1}\right) \cdots \varphi_{N}\left(z_{N}\right) q^{L_{0}-\frac{c_{v}}{24}} \xi^{\frac{H}{2}}\right) \in V_{\vec{\lambda}}^{\dagger},
$$

where $z_{1}, \ldots, z_{N}, q, \xi$ are the variables in $\mathbb{C}^{*}$ with $|q|<1, \varphi_{j}: V_{\lambda_{j}} \otimes \mathscr{H}_{\mu_{J}} \rightarrow \hat{\mathscr{H}}_{\mu_{j-1}}$ $(j=1, \ldots, N)$ are the vertex operators for some $\mu_{i}(i=0, \ldots, N)$ with $\mu_{0}=\mu_{N}=\mu, L_{0}$ is defined in (1.2.1) and $c_{v}=3 \ell /(\ell-2)$ (for the details, see $\S \S 3.4)$. It is proved that the space of restricted $N$-point functions is spanned by traces of vertex operators (Theorem 3.4.3) and hence Bernard's approach is equivalent to ours. However, the system (E1)-(E3) is not complete since it has infinite-dimensional solution space.

We will show that the integrability condition

$$
\left(E \otimes t^{-1}\right)^{\ell-2 \lambda+1}|\bar{v}(\lambda)\rangle=0
$$


for the highest weight vector $|\bar{v}(\lambda)\rangle \in \mathscr{H}_{\lambda}$ implies the differential equations (E4), which determine the $N$-point functions completely combining with (E1)-(E3).

For 1-point functions, the equation (E4) can be written down explicitly, and the system (E1)-(E4) reduces to the two equations (F1)(F2) in Theorem 4.2.4. In the simplest case, the 1-point functions are given by the characters

$$
\operatorname{Tr}_{\mathscr{H}_{\mu}} q^{L_{0}-\frac{c_{\nu}}{24}} \xi^{\frac{H}{2}}\left(\mu=0, \frac{1}{2}, \ldots, \frac{\ell}{2}\right),
$$

and our system coincide with the one obtained in [7].

Recently Felder and Wieczerkowski give a conjecture on the characterization of the restricted $N$-point functions in genus 1 by using the modular properties and certain additive conditions [9]. They confirm their conjecture in some cases by explicit calculations. We partly recover this result by solving the equation (F2) (Proposition 4.2.5). The equation (F1) can be also integrated when the dimension of the solution space is small, and we can calculate the 1-point functions explicitly.

\section{§1. Representation Theory for $\mathfrak{s l}(2, \mathbb{C})$}

For the details of the contents in this section, we refer the reader to [10].

\subsection{Integrable Highest Weight Modules}

By $\mathbb{C}[[x]]$ and $\mathbb{C}((x))$, we mean the ring of formal power series in $x$ and the field of formal Laurent series in $x$, respectively. We put $\mathfrak{g}=\mathfrak{s l}(2, \mathbb{C})$. Let $\mathfrak{h}=\mathbb{C} H$ be a Cartan subalgebra of $\mathfrak{g}$ and $():, \mathfrak{g} \times \mathfrak{g} \rightarrow \mathbb{C}$ the Cartan-Killing form normalized by the condition $(H, H)=2$. We identify the set $P_{+}$of dominant integral weights with $\frac{1}{2} \mathbb{Z}_{\geq 0}$. For $\lambda \in P_{+}$, we denote by $V_{\lambda}$ the irreducible highest weight left $\mathfrak{g}$-module with highest weight $\lambda$ and by $|v(\lambda)\rangle$ its highest weight vector.

The affine Lie algebra $\hat{\mathfrak{g}}$ associated with $\mathfrak{g}$ is defined by

$$
\hat{\mathfrak{g}}=\mathfrak{g} \otimes \mathbb{C}((x)) \oplus \mathbb{C}_{c},
$$

where $c$ is an central element of $\hat{\mathrm{g}}$ and the Lie algebra structure is given by

$$
[X \otimes f(x), Y \otimes g(x)]=[X, Y] \otimes f(x) g(x)+c \cdot(X, Y) \underset{x=0}{\operatorname{Res}}(g(x) \cdot d f(x)),
$$

for $X, Y \in \mathfrak{g}, f(x), g(x) \in \mathbb{C}((\xi))$. We use the following notations :

$$
\begin{aligned}
& X_{n}=X \otimes x^{n}, X=X_{0}, \\
& \hat{\mathfrak{g}}_{+}=\mathfrak{g} \otimes \mathbb{C}[[x]] x, \hat{\mathfrak{g}}_{-}=\mathfrak{g} \otimes \mathbb{C}\left[x^{-1}\right] x^{-1},
\end{aligned}
$$




$$
\hat{\mathfrak{p}}_{ \pm}=\hat{\mathfrak{g}}_{ \pm} \oplus \mathfrak{g} \oplus \mathbb{C}_{c}
$$

Fix a positive integer $\ell$ (called the level) and put $P_{\ell}=\left\{0, \frac{1}{2}, \ldots, \frac{\ell}{2}\right\} \subset P_{+}$. For $\lambda \in P_{\ell}$, we define the action of $\hat{\mathfrak{p}}_{+}$on $V_{\lambda}$ by $c=\ell \times i d$ and $a=0$ for all $\mathfrak{a} \in \hat{\mathfrak{g}}_{+}$, and put

$$
\mathscr{M}_{\lambda}=U(\hat{\mathfrak{g}}) \otimes_{\hat{\mathfrak{p}}_{+}} V_{\lambda} .
$$

Then $\mathscr{M}_{\lambda}$ is a highest weight left $\hat{\mathfrak{g}}$-module and it has the maximal proper submodule $\mathscr{T}_{\lambda}$, which is generated by the singular vector $E_{1}^{\ell-2 \lambda+1}|v(\lambda)\rangle$ :

$$
\mathscr{T}_{\lambda}=U\left(\hat{\mathfrak{p}}_{-}\right) E_{-1}^{\ell-2 \lambda+1}|v(\lambda)\rangle .
$$

The integrable highest weight left $\hat{\mathfrak{g}}$-module $\mathscr{H}_{\lambda}$ with highest weight $\lambda$ is defined as the quotient module $\mathscr{M}_{\lambda} / \mathscr{T}_{\lambda}$. We denote by $|\bar{v}(\lambda)\rangle$ the highest weight vector in $\mathscr{H}_{\lambda}$. We introduce the lowesd weight right $\hat{\mathrm{g}}$-module structure on

$$
\mathscr{H}_{\lambda}^{\dagger}=\operatorname{Hom}_{\mathbb{C}}\left(\mathscr{H}_{\lambda}, \mathbb{C}\right)
$$

in the usual way, and denote its lowest weight vector by $\langle\bar{v}(\lambda)|$.

\subsection{Segal-Sugawara Construction and the Filtration on $\mathscr{H}_{\lambda}$}

Fix a weight $\lambda \in P_{\ell}$. On $\mathscr{H}_{\lambda}$, elements $L_{n}(n \in \mathbb{Z})$ of the Virasoro algebra act through the Segal-Sugawara construction

$$
L_{n}=\frac{1}{2(\ell+2)} \sum_{m \in \mathbb{Z}}\left\{\circ \frac{1}{2} H_{m} H_{n-m} \circ{ }^{\circ} \circ E_{m} F_{n-m} \circ+\circ F_{m} E_{n-m} \circ\right\}
$$

where $: \circ$ denotes the standard normal ordering, and the operators $L_{n}(n \in \mathbb{Z})$ satisfy the following commutation relations:

$$
\begin{aligned}
& {\left[L_{n}, L_{m}\right]=(n-m) L_{n+m}+\frac{c_{v}}{12}\left(n^{3}-n\right) \delta_{n+m, 0},} \\
& {\left[L_{n}, X_{m}\right]=-m X_{n+m} \text { for } X \in \mathfrak{g},}
\end{aligned}
$$

where $c_{v}$ is the central charge of Virasoro algebra:

$$
c_{v}=\frac{3 \ell}{\ell+2} .
$$

Put

$$
X(z)=\sum_{n \in \mathbb{Z}} X_{n} z^{-n-1}(X \in \mathrm{g}), T(z)=\sum_{n \in \mathbb{Z}} L_{n} z^{-n-2} .
$$

The module $\mathscr{H}_{\lambda}$ has the decomposition $\mathscr{H}_{\lambda}=\bigoplus_{d \geq 0} \mathscr{H}_{\lambda}(d)$, where 


$$
\begin{aligned}
\mathscr{H}_{\lambda}(d) & =\left\{|u\rangle \in \mathscr{H}_{\lambda} ; L_{0}|u\rangle=\left(\Delta_{\lambda}+d\right)|u\rangle\right\}, \\
\Delta_{\lambda} & =\frac{\lambda(\lambda+1)}{\ell+2} .
\end{aligned}
$$

We define the filtration $\left\{\mathscr{F}_{.}\right\}$on $\mathscr{H}_{\lambda}$ by

$$
\mathscr{F}_{p} \mathscr{H}_{\lambda}=\sum_{d \leq p} \mathscr{H}_{\lambda}(d)
$$

and put $\hat{\mathscr{H}}_{\lambda}=\prod_{d \geq 0} \mathscr{H}_{\lambda}(d)$.

\subsection{The Lie Algebra $\hat{\mathbf{g}}_{\boldsymbol{N}}$}

Put $L \mathfrak{g}=\mathfrak{g} \otimes \mathbb{C}((x))$. For a positive integer $N$, we define a Lie algebra $\hat{\mathfrak{g}}_{N}$ by

$$
\hat{\mathfrak{g}}_{N}=\oplus_{j=1}^{N} L \mathfrak{g}_{(i)} \oplus \mathbb{C}_{c},
$$

where $L g_{(i)}$ denotes a copy of $L \mathfrak{g}$ and $c$ is a center. The commutation relations are given by

$$
\begin{aligned}
& {\left[\oplus_{j=1}^{N} X_{j} \otimes f_{j}, \oplus_{j=1}^{N} Y_{j} \otimes g_{j}\right]=} \\
& \quad \bigoplus_{j=1}^{N}\left[X_{j}, Y_{j}\right] \otimes f_{j} g_{j}+\sum_{j=1}^{N}\left(X_{j}, Y_{j}\right) \underset{\xi_{j}=0}{\operatorname{Res}}\left(g_{j} \cdot d f_{j}\right) \cdot c .
\end{aligned}
$$

For each $\vec{\lambda}=\left(\lambda_{1}, \ldots, \lambda_{N}\right) \in\left(P_{\ell}\right)^{N}$ a left $\hat{\mathfrak{g}}_{N}$-module $\mathscr{H}_{\vec{\lambda}}$ is defined by

$$
\mathscr{H}_{\vec{\lambda}}=\mathscr{H}_{\lambda_{1}} \otimes \cdots \otimes \mathscr{H}_{\lambda_{N}} .
$$

Similarly a right $\hat{\mathfrak{g}}_{N}$-module $\mathscr{H}_{\vec{\lambda}}^{\dagger}$ is defied by

$$
\mathscr{H}_{\vec{\lambda}}^{\dagger}=\mathscr{H}_{\lambda_{1}}^{\dagger} \hat{\otimes} \cdots \hat{\otimes} \mathscr{H}_{\lambda_{N}}^{\dagger} \cong \operatorname{Hom}_{\mathbb{C}}\left(\mathscr{H}_{\vec{\lambda}}, \mathbb{C}\right) \text {. }
$$

The $\hat{\mathrm{g}}_{N}$-action on $\mathscr{H}_{\vec{\lambda}}$ is given by

$$
\begin{aligned}
c & =\ell \cdot i d \\
\left(\oplus_{j=1}^{N} a_{j}\right)\left|u_{1} \otimes \cdots \otimes u_{N}\right\rangle & =\sum_{j=1}^{N} \rho_{j}\left(a_{j}\right)\left|u_{1} \otimes \cdots \otimes u_{N}\right\rangle
\end{aligned}
$$

for $a_{j} \in \operatorname{Lg}_{(j)}(j=1, \ldots, N)$, where we used the notations

$$
\begin{aligned}
& \left|u_{1} \otimes \cdots \otimes u_{N}\right\rangle=\left|u_{1}\right\rangle \otimes \cdots \otimes\left|u_{N}\right\rangle, \\
& \rho_{j}(a)\left|u_{1} \otimes \cdots \otimes u_{N}\right\rangle=\left|u_{1} \otimes \cdots \otimes a \cdot u_{j} \otimes \cdots \otimes u_{N}\right\rangle
\end{aligned}
$$

for $\left|u_{i}\right\rangle \in \mathscr{H}_{\lambda_{\imath}}(i=1, \ldots, N)$ and $a \in L \mathrm{~g}$. The right action on $\mathscr{H}_{\vec{\lambda}}^{\dagger}$ is defined similarly. The module $\mathscr{H}_{\vec{\lambda}}$ has the filtration induced from those of $\mathscr{H}_{\lambda_{J}}$ $(j=1, \ldots, N)$ : 


$$
\mathscr{F}_{p} \mathscr{H}_{\vec{\lambda}}=\sum_{d \leq p} \mathscr{H}_{\vec{\lambda}}(d)
$$

where

$$
\mathscr{H}_{\vec{\lambda}}(d)=\sum_{d_{1}+\cdots+d_{N}=d} \mathscr{H}_{\lambda_{1}}\left(d_{1}\right) \otimes \cdots \otimes \mathscr{H}_{\lambda_{N}}\left(d_{N}\right) .
$$

We put

$$
\begin{aligned}
& V_{\vec{\lambda}}=V_{\lambda_{1}} \otimes \cdots \otimes V_{\lambda_{N}} \cong \mathscr{H}_{\vec{\lambda}}(0), \\
& V_{\lambda}^{\dagger}=\operatorname{Hom}_{\mathbb{C}}\left(V_{\vec{\lambda}}, \mathbb{C}\right) .
\end{aligned}
$$

\section{§2. The WZNW Model in Genus 0}

In this section we review the SU(2) WZNW model on the projective line $\mathbb{P}^{1}$

\subsection{The Space of Conformal Blocks}

In this subsection we define the $N$-point functions on $\mathbb{P}^{1}$ following [13] as sections of a vector bundle on the manifold

$$
R_{N}=\left\{\left(z_{1}, \ldots, z_{N}\right) \in\left(\mathbb{C}^{*}\right)^{N} ; z_{i} \neq z_{j} \text { if } i \neq j\right\} .
$$

For a meromorphic function $f(t)$ on $\mathbb{P}^{1}$ and $w \in \mathbb{C}$, put

$$
\begin{array}{r}
X[f(t)]_{w}=\operatorname{Res}_{t=w} f(t) X(t-w) d t, \\
T\left[f(t) \frac{d}{d t}\right]_{w}=\operatorname{Res}_{t=w} f(t) T(t-w) d t .
\end{array}
$$

If $f(t)$ has an Laurent expansion $f(t)=\sum_{n \geq M} a_{n}(t-w)^{n}$ then $X[f(t)]_{w}$ is an element of $\hat{\mathrm{g}}$ given by

$$
X[f(t)]_{w}=\sum_{n \geq M} a_{n} X_{n}
$$

For $z=\left(z_{1}, \ldots, z_{N}\right) \in R_{N}$, we set

$$
\hat{\mathfrak{g}}(z)=H^{0}\left(\mathbb{P}^{1}, \mathfrak{g} \otimes \mathcal{O}_{\mathbb{P} 1}\left(* \sum_{j=1}^{N} z_{j}\right)\right) .
$$

Then we have the following injection:

$$
\hat{\mathfrak{g}}(z) \longrightarrow \hat{\mathfrak{g}}_{N},
$$




$$
X \otimes f(z) \longmapsto X[f]:=\oplus_{j=1}^{N} X[f]_{z_{j}} .
$$

Through this map we regard $\hat{\mathrm{g}}(z)$ as a subspace of $\hat{\mathrm{g}}_{N}$ and the residue theorem implies that $\hat{\mathrm{g}}(z)$ is a Lie subalgebra of $\hat{\mathrm{g}}_{N}$. We also use the following notation

$$
T[g]=\oplus_{j=1}^{N} T[g]_{z_{j}}
$$

for $g \in H^{0}\left(\mathbb{P}^{1}, \Theta_{\mathbb{P} 1}\left(* \sum_{j=1}^{N} z_{j}\right)\right)$, where $\Theta_{\mathbb{P} 1}$ denotes the sheaf of vector fields on $\mathbb{P}^{1}$.

Definition 2.1.1. For $z=\left(z_{1}, \ldots, z_{N}\right) \in R_{N}$ and $\vec{\lambda}=\left(\lambda_{1}, \ldots, \lambda_{N}\right) \in\left(P_{\ell}\right)^{N}$ we put

$$
\begin{aligned}
\mathscr{V}_{0}(z ; \vec{\lambda}) & =\mathscr{H}_{\vec{\lambda}} / \hat{\mathrm{g}}(z) \mathscr{H}_{\vec{\lambda}} \\
\mathscr{V}_{0}^{\dagger}(z ; \vec{\lambda}) & =\left\{\langle\Psi| \in \mathscr{H}_{\vec{\lambda}}^{\dagger} ;\langle\Psi| \hat{\mathfrak{g}}(z)=0\right\} \\
& \cong \operatorname{Hom}_{\mathbb{C}}\left(\mathscr{V}_{0}(z ; \vec{\lambda}), \mathbb{C}\right) .
\end{aligned}
$$

We call $\mathscr{V}_{0}^{\dagger}(z ; \vec{\lambda})$ the space of conformal blocks (or the space of vacua) in genus 0 attached to $(z ; \vec{\lambda})$.

For a vector space $V$ and a complex manifold $M$, we denote by $V[M]$ the set of multi-valued, holomorphic $V$-valued functions on $M$.

Definition 2.1.2. For $\vec{\lambda} \in\left(P_{t}\right)^{N}$, an element $\langle\Phi|$ of $\mathscr{H}_{\vec{\lambda}}^{\dagger}\left[R_{N}\right]$ is called an $N$-point function in genus 0 attached to $\vec{\lambda}$ if the following conditions are satisfied:

(A1) For each $z \in R_{N}$,

$$
\langle\Phi(z)| \in \mathscr{V}_{0}^{\dagger}(z ; \vec{\lambda})
$$

(A2) For $j=1, \ldots, N$,

$$
\partial_{z_{j}}\langle\Phi(z)|=\langle\Phi(z)| \rho_{j}\left(L_{-1}\right) .
$$

By $\mathfrak{F}_{0}(\vec{\lambda})$ we denote the set of $N$-point functions in genus 0 attached to $\vec{\lambda}$.

Remark. The condition (A1) implies the following:

(A1') For each $z \in R_{N}$,

$$
\langle\Phi(z)| T[g]=0
$$

for any $g \in H^{0}\left(\mathbb{P}^{1}, \Theta_{\mathbb{P} 1}\left(* \sum_{j=1}^{N} z_{j}\right)\right)$. 


\subsection{Restrictions of the $N$-point Functions to $V_{\vec{a}}$}

A remarkable property of the space of conformal blocks in genus 0 is the following:

Lemma 2.2.1. The composition map

$$
V_{\vec{\lambda}} \hookrightarrow \mathscr{H}_{\vec{\lambda}} \longrightarrow \mathscr{V}_{0}(z ; \vec{\lambda})
$$

is surjective. In other words, the restriction map

$$
\mathscr{V}_{0}^{\dagger}(z ; \vec{\lambda}) \longrightarrow V_{\vec{\lambda}}^{\dagger}
$$

is injective.

This lemma implies that, for an $N$-point function $\langle\Phi|$, we can calculate $\langle\Phi \mid u\rangle$ for any $|u\rangle \in \mathscr{H}_{\vec{\lambda}}$, from the data $\left\{\langle\Phi \mid v\rangle ;|v\rangle \in V_{\vec{\lambda}}\right\}$. By $\mathfrak{F}_{0}^{r}(\vec{\lambda})$ we denote the image of $\mathfrak{F}_{0}(\vec{\lambda})$ in $V_{\vec{\lambda}}^{\dagger}\left[R_{N}\right]$ under the restriction map. It is natural to ask how the set $\mathfrak{F}_{0}^{r}(\vec{\lambda})$ is characterized in $V_{\vec{\lambda}}^{\dagger}\left[R_{N}\right]$, and the answer is given as follows:

Proposition 2.2.2. [12] The space $\mathfrak{F}_{0}^{r}(\vec{\lambda})$ coincides with the solution space of the following system of equations:

(B1) For each $X \in \mathfrak{g}$,

$$
\sum_{j=1}^{N}\langle\phi(z)| \rho_{j}(X)=0 .
$$

(B2) [the Knizhnik-Zamolodchikov equations] For each $j=1, \ldots, N$,

$$
(\ell+2) \partial_{z_{j}}\langle\phi(z)|=\sum_{i \neq j}\langle\phi(z)| \frac{\Omega_{i, j}}{z_{i}-z_{j}},
$$

where

$$
\Omega_{i, j}=\frac{1}{2} \rho_{i}(H) \rho_{j}(H)+\rho_{i}(E) \rho_{j}(F)+\rho_{i}(F) \rho_{j}(E)
$$

(B3) For each $j=1, \ldots, N$,

$$
\begin{gathered}
\sum_{n_{1}+\cdots+n_{N}=\ell_{j}}\left(\begin{array}{c}
\ell_{j} \\
\vec{n}_{j}
\end{array}\right) \prod_{i \neq j}\left(z_{i}-z_{j}\right)^{-n_{i}}\left\langle\phi(z) \mid E^{n_{1}} v_{1} \otimes \cdots \otimes v\left(\lambda_{j}\right) \otimes \cdots \otimes E^{n_{N}} v_{N}\right\rangle \\
\quad=0
\end{gathered}
$$


for any $\left|v_{i}\right\rangle \in V_{\lambda_{i}}(i \neq j)$. Here $\ell_{j}=\ell-2 \lambda_{j}+1, \vec{n}_{j}=\left(n_{1}, \ldots, n_{j-1}, n_{j+1}, \ldots, n_{N}\right)$ and $\left(\begin{array}{c}\ell_{j} \\ \vec{n}_{j}\end{array}\right)$ is the multinomial coefficient.

Remark. The equation (B3) is a consequence of the integrability condition

$$
E_{-1}^{\ell-2 \lambda_{j}+1}\left|\bar{v}\left(\lambda_{j}\right)\right\rangle=0(j=1, \ldots, N)
$$

for the highest weight vector $\left|\bar{v}\left(\lambda_{j}\right)\right\rangle \in \mathscr{H}_{\lambda_{j}}$.

\subsection{Vertex Operators}

We review the description of $N$-point functions by vertex operators.

Definition 2.3.1. For $(v, \lambda, \mu) \in\left(P_{\ell}\right)^{3}$ a multi-valued, holomorphic, operator valued function $\varphi\left(z_{1}\right)$ on the manifold $\mathbb{C}^{*}=\mathbb{C} \backslash\{0\}$ is called a vertex operator of type $(v, \lambda, \mu)$, if

$$
\varphi\left(z_{1}\right): V_{\lambda} \otimes \mathscr{H}_{\mu} \longrightarrow \hat{\mathscr{H}}_{v}
$$

satisfies the following conditions:

(C1) For $X \in \mathfrak{g},|v\rangle \in V_{\lambda}$ and $m \in \mathbb{Z}$,

$$
\left[X_{m}, \varphi\left(|v\rangle ; z_{1}\right)\right]=z_{1}^{m} \varphi\left(X|v\rangle ; z_{1}\right) .
$$

(C2) For $|v\rangle \in V_{\lambda}$ and $m \in \mathbb{Z}$,

$$
\left[L_{m}, \varphi\left(|v\rangle ; z_{1}\right)\right]=z_{1}^{m}\left\{z_{1} \frac{d}{d z_{1}}+(m+1) \Delta_{\lambda}\right\} \varphi\left(|v\rangle ; z_{1}\right) .
$$

Here $\varphi\left(|u\rangle ; z_{1}\right): \mathscr{H}_{v} \rightarrow \hat{\mathscr{H}}_{\mu}$ is the operator defined by $\varphi\left(|u\rangle ; z_{1}\right)|v\rangle=$ $\varphi\left(z_{1}\right)|u \otimes v\rangle$ for $|u\rangle \in V_{\lambda}$ and $|v\rangle \in \mathscr{H}_{v}$.

For vertex operators $\varphi_{j}\left(z_{j}\right)(j=1, \ldots, N)$, the composition $\varphi_{1}\left(z_{1}\right) \cdots \varphi_{N}\left(z_{N}\right)$ makes sense for $\left|z_{1}\right|>\cdots>\left|z_{N}\right|$ and analytically continued to $R_{N}$.

Proposition 2.3.2. [12] The space $\mathfrak{F}_{0}^{r}(\vec{\lambda})$ is spanned by the following $V_{\vec{\lambda}}^{\dagger}$-valued functions:

$$
\left\langle v(0)\left|\varphi_{1}\left(z_{1}\right) \cdots \varphi_{N}\left(z_{N}\right)\right| v(0)\right\rangle,
$$

where $\varphi_{j}(j=1, \ldots, N)$ is the vertex operator of type $\left(\mu_{j-1}, \lambda_{j}, \mu_{j}\right)$ for some $\mu_{i} \in P_{\ell}(i=0, \ldots, N)$ with $\mu_{0}=\mu_{N}=0$.

Proposition 2.3.3. [12] Any nonzero vertex operator 


$$
\varphi\left(z_{1}\right): V_{\lambda} \otimes \mathscr{H}_{\mu} \longrightarrow \hat{\mathscr{H}}_{v}
$$

is uniquely extended to the operator

$$
\hat{\varphi}\left(z_{1}\right): \mathscr{M}_{\lambda} \otimes \mathscr{H}_{\mu} \longrightarrow \hat{\mathscr{H}}_{v}
$$

by the following condition:

$$
\hat{\varphi}\left(X_{n}|u\rangle ; z_{1}\right)=\operatorname{Res}_{w=z}\left(w-z_{1}\right)^{n} \hat{\varphi}\left(|u\rangle ; z_{1}\right) X(w) d w,
$$

for each $|u\rangle \in \mathscr{M}_{\lambda}, X \in \mathfrak{g}$ and $n \in \mathbb{Z}$.

Moreover, $\hat{\varphi}$ has the following properties:

$$
\begin{gathered}
\partial_{z} \hat{\varphi}\left(|u\rangle ; z_{1}\right)=\hat{\varphi}\left(L_{-1}|u\rangle ; z_{1}\right) \quad \text { for any }|u\rangle \in \mathscr{M}_{\lambda}, \\
\hat{\varphi}\left(|u\rangle ; z_{1}\right)=0 \quad \text { for any }|u\rangle \in \mathscr{T}_{\lambda}=U\left(\hat{\mathfrak{p}}_{-}\right) E_{-1}^{\ell-2 \lambda+1}|v(\lambda)\rangle .
\end{gathered}
$$

The property (2.3.3) implies that $\hat{\varphi}$ reduces to the operator

$$
\hat{\varphi}\left(z_{1}\right): \mathscr{H}_{\lambda} \otimes \mathscr{H}_{\mu} \longrightarrow \hat{\mathscr{H}}_{v} .
$$

\section{§3. The WZNW Model in Genus 1}

In this section we consider the elliptic analogue of the story in the previous section. Our aim is to embed the set of $N$-point functions in genus 1 (Definition 3.1.3) into the set of $V_{\vec{\lambda}}^{\dagger}$-valued functions, and to characterize its image by a system of differential equations. We also show that the $N$-point functions are given by the traces of vertex operators.

\subsection{Functions with Quasi-periodicity}

First, we prepare some functions for the later use. Put $D^{*}=\left\{q \in \mathbb{C}^{*} ;|q|<1\right\}$ and introduce the following functions on $\mathbb{C}^{*} \times D^{*}$ :

$$
\begin{aligned}
\Theta(z, q) & =\sum_{n \in \mathbb{Z}+\frac{1}{2}}(-1)^{n+1} q^{\frac{1}{2^{n^{2}}}} z^{n} \\
& =-\sqrt{-1} z^{\frac{1}{2}} q^{\frac{1}{8}} \prod_{n \geq 1}\left(1-q^{n}\right)\left(1-z q^{n}\right)\left(1-z^{-1} q^{n-1}\right) \\
\zeta(z, q) & =\frac{z \partial_{z} \Theta(z, q)}{\Theta(z, q)} . \\
\wp(z, q) & =-z \partial_{z} \zeta(z, q)+2 \frac{q \partial_{q} \eta(q)}{\eta(q)}
\end{aligned}
$$

where $\eta(q)$ is the Dedekind eta function 


$$
\eta(q)=q^{\frac{1}{24}} \prod_{n \geq 1}\left(1-q^{n}\right)
$$

The function $\Theta(z, q)$ satisfies the heat equation

$$
2 q \partial_{q} \Theta(z, q)=\left(z \partial_{z}\right)^{2} \Theta(z, q) .
$$

The function $\mathscr{\wp}(z, q)$ satisfies $\mathscr{P}(q z, q)=\mathscr{P}(z, q)$, and $\zeta(z, q)$ have the following quasiperiodicity:

$$
\zeta(q z, q)=\zeta(z, q)-1 .
$$

For $(z, q) \in \mathbb{C}^{*} \times D^{*}$ and $\xi \in \mathbb{C}^{*}$, we put

$$
\sigma_{ \pm}(z, q, \xi)=\frac{\Theta\left(z^{-1} \xi^{ \pm 1}, q\right) \Theta^{\prime}(1, q)}{\Theta(z, q) \Theta\left(\xi^{ \pm 1}, q\right)} .
$$

Here $\Theta^{\prime}(z, q)=z \partial_{z} \Theta(z, q)$. The function $\sigma_{ \pm}(z, q, \xi)$ have the following properties:

$$
\begin{aligned}
\sigma_{ \pm}(q z, q, \xi) & =\xi^{ \pm 1} \sigma_{ \pm}(z, q, \xi), \\
\sigma_{ \pm}\left(z^{-1}, q, \xi\right) & =-\sigma_{\mp}(z, q, \xi) .
\end{aligned}
$$

For $\zeta(z, q)$ and $\sigma_{ \pm}(z, q, \xi)$, we have the following expansion at $z=1$ :

$$
\begin{aligned}
& \zeta(z, q)=\frac{1}{z-1}+\frac{1}{2}-2 \alpha(q)(z-1)+O(z-1)^{2} \\
& \sigma_{ \pm}(z, q, \xi)=\frac{1}{z-1} \mp \zeta(\xi+q)+\frac{1}{2} \\
& \quad-\sum_{n \geq 1}\left(\frac{n \xi^{-1} q^{n}}{1-\xi^{-1} q^{n}}+\frac{n \xi q^{n}}{1-\xi q^{n}}\right)(z-1)+O(z-1)^{2}
\end{aligned}
$$

where $\alpha(q)$ is given by

$$
\alpha(q)=-\frac{q \partial_{q} \eta(q)}{\eta(q)}+\frac{1}{24} .
$$

\subsection{Twisting the Space of Conformal Blocks}

In the case of genus 1 (or $>0$ ), if we work with the formulation of [13], an $N$-point function is not determined by its restriction on $V_{\vec{\lambda}}$. In order to resolve this difficulty we "twist" the space of conformal blocks following $[1][2][6][9]$.

For $q \in D^{*}$, we consider the elliptic curve $\mathscr{E}_{q}=\mathbb{C}^{*} /\langle q\rangle$, where $\langle q\rangle$ is the infinite cyclic group of automorphisms generated by $z \mapsto q z$. We denote by 
$[z]_{q}$ the image of a point $z \in \mathbb{C}^{*}$ on $\mathscr{E}_{q}$ and put

$$
T_{N}=\left\{(z, q)=\left(z_{1}, \ldots, z_{N}, q\right) \in\left(\mathbb{C}^{*}\right)^{N} \times D^{*} ;\left[z_{i}\right]_{q} \neq\left[z_{j}\right]_{q} \text { if } i \neq j\right\} .
$$

In the following we omit the subscript $q$ in $[z]_{q}$. For $(z, q) \in T_{N}$ and $\vec{\lambda}=\left(\lambda_{1}, \ldots, \lambda_{N}\right) \in P_{\ell}$ we can define the space of conformal blocks attached to the elliptic curve $\mathscr{E}_{q}$ :

$$
\mathscr{V}_{1}^{\dagger}([z], q ; \vec{\lambda})=\left\{\langle\Psi| \in \mathscr{H}_{\vec{\lambda}}^{\dagger} ;\langle\Psi| \mathfrak{g}([z], q)=0\right\}
$$

where

$$
\hat{\mathfrak{g}}([z], q)=H^{0}\left(\mathscr{E}_{q}, \mathfrak{g} \otimes \mathcal{O}_{\mathscr{E}_{q}}\left(* \sum_{j=1}^{N}\left[z_{j}\right]\right)\right),
$$

but for our purpose we need to twist it as follows. We introduce a new variable $\xi \in \mathbb{C}^{*}$, and put

$$
\hat{\mathfrak{g}}([z], q, \xi)=\left\{a(t) \in H^{0}\left(\mathbb{C}^{*}, \mathfrak{g} \otimes \mathcal{O}_{\mathbb{C} *}\left(* \sum_{j=1}^{N} \sum_{n \in \mathbb{Z}} q^{n} z_{j}\right)\right) ; a(q t)=\xi^{\frac{H}{2}}(a(t)) \xi^{-\frac{H}{2}}\right\} .
$$

This space is regarded as the space of meromorphic sections of the $\mathrm{g}$-bundle which is twisted by $\xi^{\frac{H}{2}}$ along the cycle $\left\{[w] \in \mathscr{E}_{q} ; w \in \mathbb{R}, q \leq w<1\right\}$. For $\xi=1$, we have

$$
\hat{\mathfrak{g}}([z], q, 1)=H^{0}\left(\mathscr{E}_{q}, \mathfrak{g} \otimes \mathcal{O}_{\mathscr{E}_{q}}\left(* \sum_{j=1}^{N}\left[z_{j}\right]\right)\right) .
$$

As in the previous section we have the following injection:

$$
\begin{aligned}
\hat{\mathfrak{g}}([z], q, \xi) & \longrightarrow \hat{\mathfrak{g}}_{N} \\
X \otimes f & \longrightarrow X[f] .
\end{aligned}
$$

By this map we regard $\hat{\mathfrak{g}}([z], q, \xi)$ as a subspace of $\hat{\mathfrak{g}}_{N}$. Furthermore we can easily have the following lemma.

Lemma 3.2.1. The vector space $\hat{\mathfrak{g}}([z], q, \xi)$ is a Lie subalgebra of $\hat{\mathfrak{g}}_{N}$.

Definition 3.2.2. Put

$$
\begin{aligned}
\mathscr{V}_{1}([z], q, \xi ; \vec{\lambda}) & =\mathscr{H}_{\vec{\lambda}} / \hat{\mathfrak{g}}([z], q, \xi) \mathscr{H}_{\vec{\lambda}} \\
\mathscr{V}_{1}^{\dagger}([z], q, \xi ; \vec{\lambda}) & =\left\{\langle\Psi| \in \mathscr{H}_{\vec{\lambda}}^{\dagger} ;\langle\Psi| \hat{\mathfrak{g}}([z], q, \xi)=0\right\} \\
& \cong \operatorname{Hom}_{\mathbb{C}}\left(\mathscr{V}_{1}([z], q, \xi ; \vec{\lambda}), \mathbb{C}\right) .
\end{aligned}
$$


We call $\mathscr{V}_{1}^{\dagger}([z], q, \xi ; \vec{\lambda})$ the space of conformal blocks in genus 1 attached to $([z], q, \xi ; \vec{\lambda})$.

Following [9] [13], we define the $N$-point functions in genus 1 as follows:

Definition 3.2.3. An element $\langle\Phi|$ of $\mathscr{H}_{\vec{\lambda}}^{\dagger}\left[T_{N} \times \mathbb{C}^{*}\right]$ is called an $N$-point function in genus 1 attached to $\vec{\lambda}$ if the following conditions are satisfied:

(D1) For each $(z, q, \xi) \in T_{N} \times \mathbb{C}^{*}$,

$$
\langle\Phi(z, q, \xi)| \in \mathscr{V}_{1}^{\dagger}([z], q, \xi ; \vec{\lambda}) .
$$

(D2) For $j=1, \ldots, N$,

$$
\partial_{z_{j}}\langle\Phi(z, q, \xi)|=\langle\Phi(z, q, \xi)| \rho_{j}\left(L_{-1}\right)
$$

(D3)

$$
\left(q \partial_{q}+\frac{c_{v}}{24}\right)\langle\Phi(z, q, \xi)|=\langle\Phi(z, q, \xi)| T\left[\zeta\left(t / z_{1}, q\right) t \frac{d}{d t}\right],
$$

where $\zeta(t, q)$ is the function given by (3.1.2).

(D4)

$$
\xi \partial_{\xi}\langle\Phi(z, q, \xi)|=\langle\Phi(z, q, \xi)| \frac{1}{2} H\left[\zeta\left(t / z_{1}, q\right)\right] .
$$

We denote by $\mathfrak{F}_{1}(\vec{\lambda})$ the set of $N$-point functions attached to $\vec{\lambda}$.

Remark. (i) The condition (D1) implies the following:

(D1') For each $(z, q, \xi) \in T_{N} \times \mathbb{C}^{*}$,

$$
\langle\Phi(z, q, \xi)| T[g]=0
$$

for any $g \in H^{0}\left(\mathscr{E}_{q}, \Theta_{\mathscr{E}_{q}}\left(* \sum_{j=1}^{N} z_{j}\right)\right)=H^{0}\left(\mathscr{E}_{q}, \mathcal{O}_{\mathscr{E}_{q}}\left(* \sum_{j=1}^{N} z_{j}\right) t \frac{d}{d t}\right)$.

(ii) The equations (D1)-(D4) are compatible with each other due to (3.1.4), e.g.

$$
\begin{aligned}
& {\left[\xi \partial_{\xi}-\frac{1}{2} H\left[\zeta\left(t / z_{j}\right)\right], X[f(t, q, \xi)]\right]=} \\
& X\left[\xi \partial_{\xi} f(t, q, \xi)\right]-\frac{1}{2}[H, X]\left[\zeta\left(t / z_{j}, q\right) f(t, q, \xi)\right] \in \hat{\mathfrak{g}}([z], q, \xi),
\end{aligned}
$$

for $X[f] \in \hat{\mathfrak{g}}([z], q, \xi)$. Conversely, the compatibility condition demands 
(3.1.4) for $\zeta$.

(iii) In (D3) and (D4) we can replace $\zeta\left(t / z_{1}, q\right)$ with $\zeta\left(t / z_{j}, q\right)(j=2, \ldots, N)$ provided (D1) since

$$
\zeta\left(t / z_{1}, q\right)-\zeta\left(t / z_{j}, q\right) \in H^{0}\left(\mathscr{E}_{q}, \mathcal{O}_{\mathscr{E}_{q}}\left(* \sum_{i=1}^{N} z_{j}\right)\right) .
$$

The finite-dimensionality of the space $\mathscr{V}_{1}^{\dagger}([z], q, \xi ; \vec{\lambda})$ can be shown in a similar way as in [13]. The compatibility of (D1)-(D4) implies that there exists a vector bundle $\tilde{\mathscr{V}}_{1}^{\dagger}(\vec{\lambda})$ over a domain $U \subset T_{N} \times \mathbb{C}^{*}$ which has $\mathscr{V}_{1}^{\dagger}([z], q, \xi ; \vec{\lambda})$ as a fiber at $([z], q, \xi) \in U$, with the integrable connections defined by the differential equations (D2)-(D4). In particular the dimension of the fiber $\mathscr{V}_{1}^{\dagger}([z], q, \xi ; \vec{\lambda})$ does not depend on $([z], q, \xi)$.

\subsection{Restrictions of $N$-point Functions to $V_{\vec{\lambda}}$}

In this subsection we see that, as a consequence of the twisting, an $N$-point function in genus 1 is determined from its restriction to $V_{\vec{\lambda}}$ (Proposition 3.3.2). We also give the characterization of $N$-point functions as $V_{\vec{\lambda}}^{\dagger}$-valued functions (Theorem 3.3.4).

Lemma 3.3.1. Let $\mathscr{S}$ be the subspace of $\mathscr{H}_{\vec{\lambda}}$ spanned by the vectors

$$
\rho_{1}\left(H_{-1}\right)^{k}|v\rangle \quad\left(|v\rangle \in V_{\vec{\lambda}}, k \in \mathbb{Z}_{\geq 0}\right) .
$$

Then for $(z, q, \xi) \in T_{N} \times \mathbb{C}^{*}$ such that $\xi \neq q^{n}(n \in \mathbb{Z})$, the natural map

$$
\mathscr{P} \longrightarrow \mathscr{V}_{1}([z], q, \xi ; \vec{\lambda})
$$

is surjective. In other words the restriction map

$$
\mathscr{V}_{1}^{\dagger}([z], q, \xi ; \vec{\lambda}) \longrightarrow \operatorname{Hom}_{\mathbb{C}}(\mathscr{S}, \mathbb{C})
$$

is injective.

Proof. For $\xi \neq q^{n}(n \in \mathbb{Z})$, the space $\hat{\mathfrak{g}}([z], q, \xi)$ is spanned by the following $\mathfrak{g}$-valued functions

$$
H \otimes 1, H \otimes\left(\zeta\left(t / z_{i}, q\right)-\zeta\left(t / z_{j}, q\right)\right), H \otimes\left(t \partial_{t}\right)^{n} \wp\left(t / z_{j}, q\right),
$$

$E \otimes\left(t \partial_{t}\right)^{n} \sigma_{+}\left(t / z_{j}, q, \xi\right), F \otimes\left(t \partial_{t}\right)^{n} \sigma_{-}\left(t / z_{j}, q, \xi\right)(i, \mathrm{j}=1, \ldots, N, n=0,1, \ldots)$.

Note that for each $i=1, \ldots, N$ and $n \in \mathbb{Z}_{\geq 0}$, the function $\left(t \partial_{t}\right)^{n} \wp\left(t / z_{i}\right)$ has a pole of order $n+2$ and $\left(t \partial_{t}\right)^{n} \sigma_{ \pm}\left(t / z_{i}\right)$ has a pole of order $n+1$ at $z_{i}$. Let $\tilde{\mathscr{S}}$ be the subspace of $\mathscr{H}_{\vec{\lambda}}$ spanned by the vectors 


$$
\rho_{1}\left(H_{-1}\right)^{n_{1}} \cdots \rho_{N}\left(H_{-1}\right)^{n_{N}}|v\rangle \quad\left(n_{1}, \ldots, n_{N} \in \mathbb{Z}_{\geq 0},|v\rangle \in V_{\vec{\lambda}}\right) .
$$

Then we can show that the natural map $\tilde{\mathscr{S}} \rightarrow \mathscr{V}_{1}([z], q, \xi)$ is surjective, by induction with respect to the filtration $\{\mathscr{F}$. $\}$ on $\mathscr{H}_{\vec{\lambda}}$. Namely, for any given $|u\rangle$ of $\mathscr{F}_{p} \mathscr{H}_{\vec{\lambda}}$ with $|u\rangle \notin \tilde{\mathscr{S}}$, we can find an element $\left|u^{\prime}\right\rangle$ in $\hat{\mathfrak{g}}([z], q, \xi) \mathscr{H}_{\vec{\lambda}}$ such that

$$
|u\rangle-\left|u^{\prime}\right\rangle \in \mathscr{F}_{p-1} \mathscr{H}_{\vec{\lambda}} .
$$

Now, to prove Lemma 3.3.1, it is sufficient to note (3.2.2).

Let $\langle\Phi|$ be an $N$-point function in genus 1 and $|u\rangle$ be a vector in $\mathscr{H}_{\vec{\lambda}}$. By Lemma 3.3.1 we can express $\langle\Phi(z, q, \xi) \mid u\rangle$ as a combination of

$$
\left\langle\Phi(z, q, \xi)\left|\rho_{1}\left(H_{-1}\right)^{n}\right| v\right\rangle \quad\left(n \in \mathbb{Z}_{\geq 0},|v\rangle \in V_{\vec{\lambda}}\right) .
$$

Combining with (D4) we have the procedure to rewrite $\langle\Phi(z, q, \xi) \mid u\rangle$ as a combination of

$$
\left(\xi \partial_{\xi}\right)^{n}\langle\Phi(z, q, \xi) \mid v\rangle \quad\left(n \in \mathbb{Z}_{\geq 0},|v\rangle \in V_{\vec{\lambda}}\right) .
$$

Furthermore it is easily seen from the proof of Lemma 3.3.1 that we need finitely many data for each $|u\rangle$ :

Proposition 3.3.2. For $|u\rangle \in \mathscr{F}_{p} \mathscr{H}_{\vec{\lambda}}$, there exist functions

$$
a_{i, n}(z, q, \xi)\left(i=1, \ldots, \operatorname{dim} V_{\vec{\lambda}}, n=1, \ldots, p\right)
$$

on $T_{N} \times \mathbb{C}^{*}$ such that

$$
\langle\Phi(z, q, \xi) \mid u\rangle=\sum_{i, n} a_{i, n}(z, q, \xi)\left(\xi \partial_{\xi}\right)^{n}\left\langle\Phi(z, q, \xi) \mid b_{i}\right\rangle
$$

for any $\langle\Phi| \in \mathfrak{F}_{1}(\vec{\lambda})$, where $\left\{\left|b_{i}\right\rangle ; i=1, \ldots, \operatorname{dim} V_{\vec{\lambda}}\right\}$ is a basis of $V_{\vec{\lambda}}$.

By $\mathfrak{F}_{1}^{r}(\vec{\lambda})$ we denote the image of $\mathfrak{F}_{1}(\vec{\lambda})$ in $V_{\vec{\lambda}}^{\dagger}\left[T_{N} \times \mathbb{C}^{*}\right]$ under the restriction map to $V_{\vec{\lambda}}$, which is injective by the above proposition.

Next, as in the case of genus 0 , we consider the characterization of $\mathfrak{F}_{1}^{r}(\vec{\lambda})$ in $V_{\vec{\lambda}}^{\dagger}\left[T_{N} \times \mathbb{C}^{*}\right]$. First, we have the following.

Proposition 3.3.3. The restriction $\langle\phi|$ of an $N$-point function satisfies the following equations.

(E1)

$$
\sum_{j=1}^{N}\langle\phi(z, q, \xi)| \rho_{j}(H)=0
$$


(E2) For each $j=1, \ldots, N$,

$$
\begin{aligned}
& (\ell+2)\left(z_{j} \partial_{z_{J}}+\Delta_{\lambda_{j}}\right)(\Theta(\xi, q)\langle\phi(z, q, \xi)|)= \\
& \xi \partial_{\xi}(\Theta(\xi, q)\langle\phi(z, q, \xi)|) \rho_{j}(H)+\sum_{i \neq j} \Theta(\xi, q)\langle\phi(z, q, \xi)| \Omega_{i, j}\left(z_{j} / z_{i}, q, \xi\right),
\end{aligned}
$$

where

$$
\begin{aligned}
& \Omega_{i, j}(t, q, \xi)= \\
& \frac{1}{2} \zeta(t, q) \rho_{i}(H) \rho_{j}(H)+\sigma_{+}(t, q, \xi) \rho_{i}(F) \rho_{j}(E)+\sigma_{-}(t, q, \xi) \rho_{i}(E) \rho_{j}(F) . \\
& (\ell+2) q \partial_{q}(\Theta(\xi, q)\langle\phi(z, q, \xi)|)= \\
& \left(\xi \partial_{\xi}\right)^{2}(\Theta(\xi, q)\langle\phi(z, q, \xi)|)+\sum_{i, j=1}^{N} \Theta(\xi, q)\langle\phi(z, q, \xi)| \Lambda_{i, j}\left(z_{i} / z_{j}, q, \xi\right) .
\end{aligned}
$$

Here

$$
\begin{aligned}
& \Lambda_{i, j}(t, q, \xi)=\frac{1}{4}\left(\zeta(t, q)^{2}-\wp(t, q)\right) \rho_{i}(H) \rho_{j}(H) \\
& +\omega_{+}(t, q, \xi) \rho_{i}(E) \rho_{j}(F)+\omega_{-}(t, q, \xi) \rho_{i}(F) \rho_{j}(E)
\end{aligned}
$$

where $\omega_{ \pm}(t, q, \xi)$ denote the functions defined by

$$
\omega_{ \pm}(t, q, \xi)=\frac{1}{2}\left\{\partial_{t} \sigma_{ \pm}(t, q, \xi)+(\zeta(t, q) \pm \zeta(\xi, q)) \sigma_{ \pm}(t, q, \xi)\right\},
$$

which are holomorphic at $t=1$.

For the proof of Proposition 3.3.3, we refer the reader to [9].

Remark. The equation (E2) is derived by Bernard as a equation for the trace of the vertex operators (see $\S 3.4$ ), he also derived (E3) in a special case. The equations (E2)(E3) are called the Knizhnik-Zamolodchikov-Bernard (KZB) equations in [8] [9].

Note that the system of equations (E1)-(E3) is not holonomic since we have $j+2$ parameters $z_{1}, \ldots, z_{N}, q, \xi$, but have only $j+1$ differential equations, which are compatible each other.

The differential equations (E2) and (E3) are of order 1 with respect to $z_{j}$ $(j=1, \ldots, N)$ and $q$ respectively. Hence to characterize $\mathfrak{F}_{1}^{r}(\vec{\lambda})$ in $V_{\vec{\lambda}}\left[T_{N} \times \mathbb{C}^{*}\right]$, it is sufficient to obtain equations which determine the $\xi$-dependence of the restricted $N$-point functions and they are obtained as follows.

Let $\langle\Phi|$ be an $N$-point function and $\langle\phi|$ its restriction to $V_{\vec{\lambda}}$. We put $\mathscr{M}_{\vec{\lambda}}^{\dagger}=\operatorname{Hom}_{\mathbb{C}}\left(\mathscr{M}_{\lambda_{1}} \otimes \cdots \otimes \mathscr{M}_{\lambda_{N}} \mathbb{C}\right)$ and regard $\langle\Phi|$ as an $\mathscr{M}_{\vec{\lambda}}^{\dagger}$-valued function. 
Then as a special case of integrability condition, we have for each non negative integer $k$

$$
\left\langle\Phi \mid v_{1} \otimes \cdots \otimes F^{k} E_{-1}^{\ell-2 \lambda_{j}+1} v\left(\lambda_{j}\right) \otimes \cdots \otimes v_{N}\right\rangle=0
$$

for any $\left|v_{i}\right\rangle \in V_{\lambda_{i}}(i \neq j)$, where $\left|v\left(\lambda_{j}\right)\right\rangle$ denotes the highest weight vector in $\mathscr{M}_{\lambda_{j}}$.

On the other hand, by Proposition 3.3.2 we can rewrite the left hand side of (3.3.1) as a combination of

$$
\left(\xi \partial_{\xi}\right)^{n}\langle\Phi \mid v\rangle=\left(\xi \partial_{\xi}\right)^{n}\langle\phi \mid v\rangle \quad\left(n=0,1, \ldots, \ell-2 \lambda_{j}+1,|v\rangle \in V_{\vec{\lambda}}\right) .
$$

Now the equality (3.3.1) implies the differential equation for $\langle\phi|$ with respect to $\xi$ of order at most $\ell-2 \lambda+1$. We denote this differential equation by

$$
\left\langle\phi \mid v_{1} \otimes \cdots \otimes F^{k} E_{-1}^{\ell-2 \lambda_{j}+1} v\left(\lambda_{j}\right) \otimes \cdots \otimes v_{N}\right\rangle=0 .
$$

Theorem 3.3.4. The space $\mathfrak{F}_{1}^{r}(\vec{\lambda})$ coincides with the solution space of the system of equations (E1)-(E4), where (E4) is given by

(E4) For each $j=1, \ldots, N$ and nonnegative integer $k \leq \sum_{i=1}^{N} \lambda_{i}+\ell-$ $2 \lambda_{j}+1$

$$
\left\langle\phi(z, q, \xi) \mid v_{1} \otimes \cdots \otimes F^{k} E_{-1}^{\ell-2 \lambda_{J}+1} v\left(\lambda_{j}\right) \otimes \cdots \otimes v_{N}\right\rangle=0,
$$

for any $\left|v_{i}\right\rangle \in V_{\lambda_{1}}(i \neq j)$.

Proof. It is enough to prove that the dimension of the solution space of the system (E1)-(E4) is not larger than $\operatorname{dim}_{\mathbb{C}} \mathfrak{F}_{1}(\vec{\lambda})=\operatorname{dim}_{\mathbb{C}} \mathfrak{\mho}_{1}^{r}(\vec{\lambda})$.

Fix $(z, q) \in T_{N}$ and let $\langle\phi(\xi)|=\langle\phi(z, q, \xi)|$ be a $V_{\vec{\lambda}}^{\dagger}$-valued function on $\mathbb{C}^{*}$ which satisfies (E1) and (E4). From $\langle\phi(\xi)|$, we construct an element $\langle\Phi(\xi)|$ of $\mathscr{H}_{\vec{\lambda}}^{\dagger}\left[\mathbb{C}^{*}\right]$ which satisfies

$$
\begin{aligned}
& \langle\Phi(\xi) \mid v\rangle=\langle\phi(\xi) \mid v\rangle \text { for }|v\rangle \in V_{\vec{\lambda}}, \\
& \langle\Phi(\xi)| \in \mathscr{V}_{1}^{\dagger}([z], q, \xi ; \vec{\lambda}) \text { for each } \xi \in \mathbb{C}^{*}, \\
& \xi \partial_{\xi}\langle\Phi(\xi)|=\langle\Phi(\xi)| \frac{1}{2} H\left[\zeta\left(t / z_{1}\right)\right],
\end{aligned}
$$

through the steps below. This means that we have the injective homomorphism from the solution space of (E1)-(E4) to the space of functions on $\mathbb{C}^{*}$ satisfying (ii) and (iii), and such space has the same dimension as $\mathscr{V}_{1}^{\dagger}([z], q, \xi ; \vec{\lambda})$.

Step 1. We extend $\langle\phi(\xi)|$ to a $\mathscr{M}_{\vec{\lambda}}^{\dagger}$-valued function $\langle\Phi(\xi)|$ on $\mathbb{C}^{*}$ in 
such a way that $\langle\Phi(\xi)|=\langle\Phi(z, q, \xi)|$ satisfies

$$
\begin{aligned}
& \langle\Phi(\xi)| X[f]=0 \text { for } X[f] \in \hat{\mathfrak{g}}([z], q, \xi), \\
& \langle\Phi(\xi)| \frac{1}{2} H\left[\zeta\left(t / z_{1}, q\right)\right]=\xi \partial_{\xi}\langle\Phi(\xi)| .
\end{aligned}
$$

The well-definedness is proved by induction with respect to the filtration $\left\{\mathscr{F}_{0}\right\}$ using Lemma 3.2.1 and the compatibility condition (3.2.1).

Step 2. We show that $\langle\Phi(\xi)|$ belongs to $\mathscr{H}_{\vec{\lambda}}^{\dagger}$, that is,

$$
\left\langle\Phi(\xi) \mid u_{1} \otimes \cdots \otimes a \cdot E_{-1}^{\ell-2 \lambda+1} v\left(\lambda_{j}\right) \otimes \cdots \otimes u_{N}\right\rangle=0
$$

for any $j=1, \ldots, N,\left|u_{i}\right\rangle \in \mathscr{M}_{\lambda_{l}}$ and $a \in U\left(\hat{\mathfrak{p}}_{-}\right)$. This is reduced to (E4) also by induction.

In the case of $N=1$ we can write down the differential equations (E4) explicitly as we will see in $\S 4$.

\subsection{Sewing Procedure}

In this subsection we show that the $N$-point functions in genus 1 are given by the traces of vertex operators and hence Bernard's approach is equivalent to ours. For this purpose we construct an $N$-point function in genus 1 from an $N+2$-point function in genus 0 . This construction is known as the sewing procedure.

Fix $\mu \in P_{\ell}$ and $\vec{\lambda}=\left(\lambda_{1}, \ldots, \lambda_{N}\right) \in\left(P_{\ell}\right)^{N}$, and consider a sequence of vertex operators $\varphi_{j}\left(z_{j}\right): V_{\lambda_{j}} \otimes \mathscr{H}_{\mu_{j}} \rightarrow \hat{\mathscr{H}}_{\mu_{j-1}}$ for some $\mu_{j-1}, \mu_{j} \in P_{\ell}$ with $\mu_{0}=\mu_{N}=\mu$. For $|u\rangle=\left|u_{1} \otimes \cdots \otimes u_{N}\right\rangle \in \mathscr{H}_{\vec{\lambda}}$, we put

$$
\Phi_{0}(|u\rangle ; z)=\hat{\varphi}_{1}\left(\left|u_{1}\right\rangle ; z_{1}\right) \hat{\varphi}_{2}\left(\left|u_{2}\right\rangle ; z_{2}\right) \cdots \hat{\varphi}_{N}\left(\left|u_{N}\right\rangle ; z_{N}\right): \mathscr{H}_{\mu} \longrightarrow \hat{\mathscr{H}}_{\mu},
$$

where $\hat{\varphi}_{j}\left(z_{j}\right)$ means the extended vertex operator in the sense of Proposition 2.3.3. We define a $\mathscr{H}_{\vec{\lambda}}^{\dagger}$-valued function on $T_{N} \times \mathbb{C}^{*}$ by

$$
\left\langle\Phi_{1}(z, q, \xi) \mid u\right\rangle=\operatorname{Tr}_{\mathscr{H}_{\mu}}\left(\Phi_{0}(|u\rangle ; z) q^{L_{0}-\frac{c_{v}}{24}} \xi^{\frac{H}{2}}\right)
$$

for $|u\rangle \in \mathscr{H}_{\vec{\lambda}}$.

Proposition 3.4.1. The element $\left\langle\Phi_{1}\right|$ of $\mathscr{H}_{\vec{\lambda}}\left[T_{N} \times \mathbb{C}^{*}\right]$ defined by (3.4.1) is an $N$-point function in genus 1.

Proof. First we prove that $\left\langle\Phi_{1}\right|$ satisfies the condition (D1).

Fix any $X \otimes f \in \hat{\mathfrak{g}}([z], q, \xi ; \vec{\lambda})$ and $|u\rangle \in \mathscr{H}_{\vec{\lambda}}$, and put 


$$
\left\langle\Phi_{1}|X(t)| u\right\rangle d t=\operatorname{Tr}_{\mathscr{H}_{\mu}} \Phi_{0}(|u\rangle ; z) X(t) q^{L_{0}-\frac{c_{v}}{24}} \xi^{\frac{H}{2}} d t .
$$

This is a holomorphic 1 -form on $\mathbb{C}^{*} \backslash\left\{q^{n} z_{j} \in \mathbb{C}^{*} ; n \in \mathbb{Z}, j=1, \ldots, N\right\}$. Then by (2.3.1), what we should show is the following.

$$
\sum_{j=1}^{N} \operatorname{Res}_{t=z_{J}} f(t)\left\langle\Phi_{1}|X(t)| u\right\rangle d t=0 .
$$

We have

$$
\begin{aligned}
f(t)\left\langle\Phi_{1}|X(t)| u\right\rangle d t & =f(t) \operatorname{Tr}_{\mathscr{H}_{\mu}} X(t) \Phi_{0}(|u\rangle ; z) q^{L_{0}-\frac{c_{\nu}}{24}} \xi^{\frac{H}{2}} d t \\
& =f(t) \operatorname{Tr}_{\mathscr{H}_{\mu}} \Phi_{0}(|u\rangle ; z) q^{L_{0}-\frac{c_{\nu}}{24} \xi^{\frac{H}{2}} X(t) d t} \\
& =f(q t) \operatorname{Tr}_{\mathscr{H}_{\mu}} \Phi_{0}(|u\rangle ; z) X(q t) q^{L_{0}-\frac{c_{\nu}}{24}} \xi^{\frac{H}{2}} d(q t) \\
& =f(q t)\left\langle\Phi_{1}|X(q t)| u\right\rangle d(q t),
\end{aligned}
$$

where we used the commutativity of vertex operators and currents, and

$$
f(t) \xi^{\frac{H}{2}}(X(t)) \xi^{-\frac{H}{2}}=f(q t) X(t), q^{L_{0}}(X(t)) q^{-L_{0}}=X(q t) q .
$$

Therefore we have $f(t)\left\langle\Phi_{1}|X(t)| u\right\rangle d t \in H^{0}\left(\mathscr{E}_{q}, \omega_{\mathscr{E}_{q}}\left(\sum_{j=1}^{N} *\left[z_{j}\right]\right)\right)$, where $\omega_{\mathscr{E}_{q}}$ denotes the sheaf of 1 -forms on $\mathscr{E}_{q}$. This implies (3.4.2).

Next we prove that $\langle\Phi|$ satisfies the equation (D2)-(D4). It is obvious that $\langle\Phi|$ satisfies (D2) from (2.3.2). We give a proof of (D4). The equation (D3) is proved in a similar way. We chose $(z, q)$ from the region $1>\left|z_{1}\right|>\left|z_{2}\right|>\cdots>\left|z_{N}\right|>|q|$, where $\left\langle\Phi_{1}\right|$ is a convergent power series. Let $Z_{r}=\{|w|=r\}$ be a cycle with anticlockwise orientation. We have

$$
\begin{aligned}
2 \pi & \sqrt{-1}\left\langle\Phi_{1}\left|H\left[\zeta\left(t / z_{1}\right)\right]\right| u\right\rangle \\
= & \operatorname{Tr}_{\mathscr{H}_{\mu}} \int_{Z_{1}} \zeta\left(t / z_{1}\right) H(t) \Phi_{0}(|u\rangle ; z) q^{L_{0}-\frac{c_{u}}{24}} \xi^{\frac{H}{2}} d t \\
& -\operatorname{Tr}_{\mathscr{H}_{\mu}} \int_{Z_{q}} \zeta\left(t / z_{1}\right) \Phi_{0}(|u\rangle ; z) H(t) q^{L_{0}-\frac{c_{v}}{24}} \xi^{\frac{H}{2}} d t \\
= & \operatorname{Tr}_{\mathscr{H}_{\mu}}\left\{\int_{Z_{q}} \zeta\left(q^{-1} t / z_{1}\right)-\int_{Z_{q}} \zeta\left(t / z_{1}\right)\right\} \Phi_{0}(|u\rangle ; z) H(t) q^{L_{0}-\frac{c_{\nu}}{24}} \xi^{\frac{H}{2}} d t .
\end{aligned}
$$

By $\zeta(t)=\zeta\left(q^{-1} t\right)-1$, we conclude

$$
\left\langle\Phi_{1}\left|H\left[\zeta\left(t / z_{1}\right)\right]\right| u\right\rangle
$$




$$
\begin{aligned}
& =\frac{1}{2 \pi \sqrt{-1}} \operatorname{Tr}_{\mathscr{H}_{\mu}} \int_{Z_{q}} \Phi_{0}(|u\rangle ; z) H(t) q^{L_{0}-\frac{c_{v}}{24}} \xi^{\frac{H}{2}} d t \\
& =\operatorname{Tr}_{\mathscr{H}_{\mu}}\left(\Phi_{0}(|u\rangle ; z) H q^{L_{0}-\frac{c_{v}}{24}} \xi^{\frac{H}{2}}\right) .
\end{aligned}
$$

This proves (D4).

By Proposition 3.4.1 we have the mapping from $\mathfrak{F}_{0}(\mu, \vec{\lambda}, \mu)$ to $\mathfrak{F}_{1}(\vec{\lambda})$. We denote this mapping by $\jmath_{\mu}$. The following proposition follows from "the factorization property" proved in [13].

Proposition 3.4.2. The following map is bijective

$$
\bigoplus_{\mu \in P_{\ell}} \Im_{\mu}: \underset{\mu \in P_{\ell}}{\bigoplus} \mathfrak{F}_{0}(\mu, \vec{\lambda}, \mu) \longrightarrow \mathfrak{F}_{1}(\vec{\lambda})
$$

By Proposition 2.3.2 and Proposition 3.4.2 we get the following.

Theorem 3.4.3. The space $\mathfrak{F}_{1}^{r}(\vec{\lambda})$ is spanned by the functions

$$
\operatorname{Tr}_{\mathscr{H}_{\mu}} \varphi_{1}\left(z_{1}\right) \cdots \varphi_{N}\left(z_{N}\right) q^{L_{0}-\frac{c_{\nu}}{24}} \xi^{\frac{H}{2}}
$$

where $\varphi_{j}\left(z_{j}\right)(j=1, \ldots, N)$ is the vertex operator of type $\left(\mu_{j-1}, \lambda_{j}, \mu_{j}\right)$ for some $\mu_{i} \in P_{\ell}(i=1, \ldots, N+1)$ with $\mu:=\mu_{0}=\mu_{N}$.

Remark. The integral representations of the above functions are obtained in [3].

\section{§4. Explicit Formulas for 1-Point Functions in Genus 1}

In this section, we see how the system (E1)-(E4) determine the 1-point function explicitly (Theorem 4.2.4). We also solve the system in a few cases in the last subsection.

\subsection{The 1-Point Functions in Genus 1}

Fix a weight $\lambda$ and consider the set $\mathfrak{F}_{1}^{r}(\lambda)$ of restricted 1-point functions in genus 1 , which is, by Theorem 3.4 .3 , spanned by the following $V_{\lambda}^{\dagger}$-valued functions :

$$
\left\langle\phi_{\mu}\left(z_{1}, q, \xi\right)\right|:=\operatorname{Tr}_{\mathscr{H}_{\mu}} \varphi\left(z_{1}\right) q^{L_{0}-\frac{c_{\nu}}{24}} \xi^{\frac{H}{2}}\left(\mu \in P_{\ell}\right),
$$

where $\varphi\left(z_{1}\right)$ is the vertex operator of type $(\mu, \lambda, \mu)$. We put 


$$
L=\ell-2 \lambda \text {. }
$$

Note that a nonzero vertex operator of type $(\mu, \lambda, \mu)$ exists if and only if $\lambda$ and $\mu$ satisfy

$$
\lambda \in \mathbb{Z}, \frac{\lambda}{2} \leq \mu \leq \frac{\lambda+L}{2}
$$

and the vertex operators are unique up to constant multiples. In particular we have

$$
\operatorname{dim}_{\mathbb{C}} \mathfrak{F}_{1}(\lambda)=L+1 .
$$

As we have seen in Theorem 3.3.4, the restrictions of 1-point functions $\langle\phi|$ are characterized by (E1)-(E4). The equation (E2) now implies

$$
\left\langle\phi\left(z_{1}, q, \xi\right)\right|=z_{1}^{-\Delta \lambda}\langle\phi(1, q, \xi)| .
$$

Hence in the following we specialize $z_{1}=1$ and put

$$
\langle\phi(\xi, q)|=\langle\phi(1, q, \xi)| .
$$

The condition (E1) implies

$$
\langle\phi(\xi, q) \mid v\rangle=0
$$

unless $|v\rangle \in \mathbb{C}\left|0_{\lambda}\right\rangle$, where $\left|0_{\lambda}\right\rangle$ is the weight 0 vector in $V_{\lambda}$ defined by

$$
\left|0_{\lambda}\right\rangle=\frac{1}{\lambda !} F^{\lambda}|v(\lambda)\rangle
$$

We put

$$
\phi_{\mu}(\xi, q)=\left\langle\phi_{\mu}(\xi, q) \mid 0_{\lambda}\right\rangle=\operatorname{Tr}_{\mathscr{H}_{\mu}} \varphi_{\mu}\left(|0\rangle_{\lambda} ; 1\right) q^{L_{0}-\frac{c_{v}}{24}} \xi^{\frac{H}{2}},
$$

and identify $\mathfrak{F}_{1}^{r}(\lambda)$ with the space spanned by the following function:

$$
\phi_{\mu}(\xi, q) \mu=\frac{\lambda}{2}, \frac{\lambda+1}{2}, \ldots, \frac{\lambda+L}{2} .
$$

From the equation (E3) we immediately have the following heat equation.

Proposition 4.1.1. For $\phi \in \mathfrak{F}_{1}^{r}(\lambda)$,

$$
\begin{aligned}
& (\ell+2) q \partial_{q}(\Theta(\xi, q) \phi(\xi, q))= \\
& \left\{\left(\xi \partial_{\xi}\right)^{2}-\lambda(\lambda+1)\left(\wp(\xi, q)-2 \frac{q \partial_{q} \eta(q)}{\eta(q)}\right)\right\}(\Theta(\xi, q) \phi(\xi, q)) .
\end{aligned}
$$


Remark. The heat equations for 1-point functions are studied by Etingof and Kirillov in more general cases: $\mathfrak{g}=\mathfrak{s l}(n, \mathbb{C}), V_{\lambda}=S^{\lambda} \mathbb{C}^{n}(\lambda \in \mathbb{Z})$, where $S^{\lambda}$ denotes $\lambda$-th symmetric product and $\mathbb{C}^{n}$ the defining representation of $\mathfrak{g}[5]$.

\subsection{The Differential Equation with Respect to $\xi$}

This subsection is devoted to write down differential equations for by $\phi \in \mathfrak{F}_{1}^{r}(\lambda)$ derived from (E4):

$$
\left\langle\phi\left|F^{k} E_{-1}^{L+1}\right| v(\lambda)\right\rangle=0 \quad(0 \leq k \leq \lambda+L+1) .
$$

Among them the only nontrivial equality is the following:

$$
\left\langle\phi\left|F^{\lambda+L+1} E_{-1}^{L+1}\right| v(\lambda)\right\rangle=0,
$$

because other equalities fall into trivial by (E1).

To rewrite (4.2.1) as a differential equation with respect to $\xi$, we consider the following set vectors in $\mathscr{M}_{\lambda}$

$$
\left\{\left|u^{k}\right\rangle=\frac{1}{(\lambda+k) !} F^{\lambda+k} E_{-1}^{k}|v(\lambda)\rangle ; k=0,1, \ldots\right\} .
$$

Note that $\left|u^{0}\right\rangle=\left|0_{\lambda}\right\rangle$. The following lemma plays a key role in the following discussions.

Lemma 4.2.1. For $k \in \mathbb{Z}_{\geq 0}$, we have

$$
\begin{aligned}
& \frac{1}{2} H[\zeta(z, q)]\left|u^{k}\right\rangle \equiv \\
-\frac{1}{2}\left|u^{k+1}\right\rangle+(k+\lambda) \zeta(\xi, q)\left|u^{k}\right\rangle+k(L-k+1) \beta(\xi, q)\left|u^{k-1}\right\rangle & \bmod \hat{\mathfrak{g}}([\mathbb{z}], q, \xi) \mathscr{M}_{\lambda},
\end{aligned}
$$

where $\beta(\xi, q)$ is given by

$$
\beta(\xi, q)=\frac{q \partial_{q} \Theta(\xi, q)}{\Theta(\xi, q)}-3 \frac{q \partial_{q} \eta(q)}{\eta(q)}
$$

Proof. First we have

$$
\frac{1}{2} H[\zeta(z, q)]\left|u^{k}\right\rangle=\frac{1}{2}\left(H_{-1}-2 \alpha(q) H_{1}\right)\left|u^{k}\right\rangle
$$

since $H_{n}\left|u^{k}\right\rangle=0$ for $n=0$ and $n \geq 2$. The second term in the right hand side of (4.2.4) can be calculated as follows: 


$$
H_{1}\left|u^{k}\right\rangle=-2 k(L-k+1)\left|u^{k-1}\right\rangle
$$

This follows from

$$
\begin{aligned}
& H_{1} F^{\lambda+k} E_{-1}^{k}|v(\lambda)\rangle=\left[H_{1}, F^{\lambda+k} E_{-1}^{k}\right]|v(\lambda)\rangle \\
& \quad=-2(\lambda+k) F^{\lambda+k-1} F_{1} E_{-1}^{k}|v(\lambda)\rangle \\
& \quad=-2 k(L-k+1)(\lambda+k) F^{\lambda+k-1} E_{-1}^{k-1}|v(\lambda)\rangle .
\end{aligned}
$$

To rewrite the first term in the right hand side of (4.2.4) as a linear combination of $\left|u^{n}\right\rangle$ 's, we use the equality

$$
\begin{aligned}
{\left[E_{-1}, F^{\lambda+k+1} E_{-1}^{k}\right]=} & (\lambda+k+1) H_{-1} F^{\lambda+k} E_{-1}^{k} \\
& +(\lambda+k)(\lambda+k+1) F_{-1} F^{\lambda+k-1} E_{-1}^{k} .
\end{aligned}
$$

Thus we have

$$
\begin{aligned}
(\lambda+k+1) H_{-1} F^{\lambda+k} E_{-1}^{k}|v(\lambda)\rangle & \\
= & E_{-1} F^{\lambda+k+1} E_{-1}^{k}|v(\lambda)\rangle-F^{\lambda+k+1} E_{-1}^{k+1}|v(\lambda)\rangle \\
& \quad-(\lambda+k)(\lambda+k+1) F_{-1} F^{\lambda+k-1} E_{-1}^{k}|v(\lambda)\rangle \\
\equiv & E\left[(z-1)^{-1}-\sigma_{+}(z)\right] F^{\lambda+k+1} E_{-1}^{k}|v(\lambda)\rangle-F^{\lambda+k+1} E_{-1}^{k+1}|v(\lambda)\rangle \\
& -(\lambda+k)(\lambda+k+1) F\left[(z-1)^{-1}-\sigma_{-}(z)\right] F^{\lambda+k-1} E_{-1}^{k}|v(\lambda)\rangle \\
& \bmod \hat{\mathfrak{g}}_{\xi}\left(\mathfrak{X}_{q}\right) \mathscr{M}_{\lambda} .
\end{aligned}
$$

Since we can easily check

$$
\begin{aligned}
& E_{n} F^{\lambda+k+1} E_{-1}^{k}|v(\lambda)\rangle=0, \\
& F_{n} F^{\lambda+k-1} E_{-1}^{k}|v(\lambda)\rangle=0
\end{aligned}
$$

for $n \geq 2$, we have

$$
\begin{aligned}
& E\left[(z-1)^{-1}-\sigma_{+}(z)\right] F^{\lambda+k+1} E_{-1}^{k}|v(\lambda)\rangle= \\
& \quad+\left(\zeta(\xi, q)-\frac{1}{2}\right) E F^{\lambda+k+1} E_{-1}^{k}|v(\lambda)\rangle+(\beta(\xi, q)-2 \alpha(q)) E_{1} F^{\lambda+k+1} E_{-1}^{k}|v(\lambda)\rangle
\end{aligned}
$$

and have a similar equality for $F\left[(z-1)^{-1}-\sigma_{-}(z)\right] F^{\lambda+k-1} E_{-1}^{k}|v(\lambda)\rangle$. Here we used (3.1.8). By some more straightforward calculations, we have (4.2.2).

For $\langle\Phi| \in \mathfrak{F}_{1}(\lambda)$, we put

$$
\vec{\Phi}={ }^{t}\left(\left\langle\Phi \mid u^{0}\right\rangle, \ldots,\left\langle\Phi \mid u^{L}\right\rangle\right) .
$$

Then by $\left\langle\Phi \mid u^{L+1}\right\rangle=0$ and Lemma 4.2 .1 , we obtain the following differential equation for $\vec{\Phi}$. 
Proposition 4.2.2. For $\langle\Phi| \in \mathfrak{F}_{1}(\lambda)$, we have

$$
\xi \partial_{\xi} \vec{\Phi}(\xi, q)=\mathscr{A}_{L+1}(\xi, q) \vec{\Phi}(\xi, q)+\lambda \zeta(\xi, q) \vec{\Phi}(\xi, q)
$$

Here. $\mathscr{A}_{L+1}$ is an $(L+1) \times(L+1)$ tri-diagonal matrix given by

$$
\mathscr{A}_{L+1}=
$$

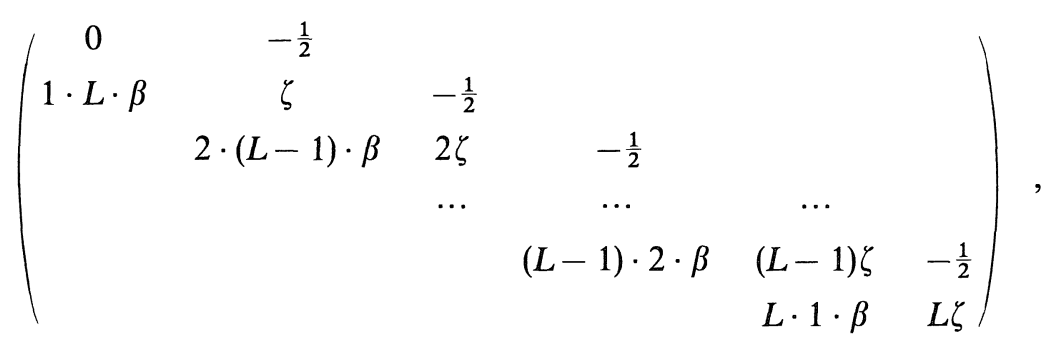

where the functions $\zeta(\xi, q)$ and $\beta(\xi, q)$ are given by (3.1.2) and (4.2.3).

It is remarkable that the equation (4.2.7) can be written in the following form :

$$
\xi \partial_{\xi}\left(\Theta^{-\lambda} \vec{\Phi}\right)=\mathscr{A}_{L+1}\left(\Theta^{-\lambda} \vec{\Phi}\right)
$$

By Proposition 4.2.2, we can write $\left\langle\Phi \mid u^{k}\right\rangle$ as a combination of differentials of $\phi:=\left\langle\Phi \mid u^{0}\right\rangle$ with respect to $\xi$; e.g.

$$
\begin{aligned}
\left\langle\Phi \mid u^{1}\right\rangle & =-2 \xi \partial_{\xi} \phi, \\
\left\langle\Phi \mid u^{2}\right\rangle & =-2\left(\xi \partial_{\xi}-\zeta\right)\left\langle\Phi \mid u^{1}\right\rangle-2 L \beta \phi \\
& =4\left(\xi \partial_{\xi}-\zeta\right) \xi \partial_{\xi} \phi-2 L \beta \phi, \\
& \text { etc } . .
\end{aligned}
$$

In general, we have the following lemma by (4.2.9) and simple calculations.

Lemma 4.2.3. For $k=1, \ldots, L+1$, we have

$$
\Theta^{-\lambda}\left\langle\Phi \mid u^{k}\right\rangle=(-2)^{k} \operatorname{Det}\left[\xi \partial_{\xi} \cdot I_{k}-\mathscr{A}_{L+1}^{(k)}\right]\left(\Theta^{-\lambda} \phi\right),
$$

where $I_{k}$ is the $k \times k$-identity matrix and $\mathscr{A}_{L+1}^{(k)}$ is the $k \times k$-matrix given by the first $k \times k$ block of $\mathscr{A}_{L+1}$ :

$$
\mathscr{A}_{L+1}^{(k)}=\left(\begin{array}{ccccc}
0 & -\frac{1}{2} & & & \\
L \beta & \zeta & -\frac{1}{2} & & \\
& 2(L-1) \beta & 2 \zeta & -\frac{1}{2} & \ldots \\
& & \cdots & \cdots & (k-1) \zeta
\end{array}\right) .
$$


Here, for an $n \times n$-matrix $A=\left(a_{i, j}\right)$ with elements in some, possibly noncommutative, ring, Det $A$ is defined inductively as follows:

Det $A=a_{1,1}$ for $n=1$,

Det $A=\operatorname{Det} A_{1,1} \cdot a_{1,1}-\operatorname{Det} A_{1,2} \cdot a_{1,2}+\cdots+(-1)^{n-1}$ Det $A_{1, n} \cdot a_{1, n}$,

where $A_{i, j}$ is the matrix given by removing the $i$-th row and $j$-th column from A.

Through this lemma, we can rewrite (4.2.1) explicitly as a differential equation for $\phi \in \mathfrak{F}_{1}^{r}(\lambda)$ of order $L+1$ with respect to $\xi$. Combining with (4.1.1) we have the following.

Theorem 4.2.4. The space $\mathfrak{F}_{1}^{r}(\lambda)$ coincides with the solution space of the following system of differential equations.

$$
\begin{aligned}
& (\ell+2) q \partial_{q}\left(\Theta(\xi, q)^{-\lambda} \phi(\xi, q)\right)= \\
& \left\{\left(\xi \partial_{\xi}\right)^{2}+2(\lambda+1) \zeta(\xi, q) \xi \partial_{\xi}-L(\lambda+1) \frac{q \partial_{q} \Theta(\xi, q)}{\Theta(\xi, q)}\right\}\left(\Theta(\xi, q)^{-\lambda} \phi(\xi, q)\right) .
\end{aligned}
$$

$$
\text { Det }\left[\xi \partial_{\xi} \cdot I_{L+1}-\mathscr{A}_{L+1}(\xi, q)\right]\left(\Theta(\xi, q)^{-\lambda} \phi(\xi, q)\right)=0 \text {. }
$$

Remark. (i) It is easy to see directly that the solution space of (F1)(F2) is $(L+1)$-dimensional.

(ii) For $\lambda=0$, the vertex operator $\varphi_{\mu}\left(|0\rangle_{0} ; z\right)$ is equal to the identity operator on $\mathscr{H}_{\mu}$ up to a constant multiple. Thus the 1-point function $\phi_{\mu}$ is nothing but the character

$$
\chi_{\mu}^{(\ell)}(\xi, q)=\operatorname{Tr}_{\mathscr{H}_{\mu}} q^{L_{0}-\frac{c_{\nu}}{24}} \xi^{\frac{H}{2}}=\frac{\Theta_{2 \mu+1, \ell+2}(\xi, q)-\Theta_{-2 \mu-1, \ell+2}(\xi, q)}{\sqrt{-1} \Theta(\xi, q)},
$$

where $\Theta_{m, k}(\xi, q)$ is the theta function of level $k$ defined by

$$
\Theta_{m, k}(\xi, q)=\sum_{n \in \mathbb{Z}+\frac{m}{2 k}} q^{k n^{2}} \xi^{k n}
$$

In the case of $\ell=1,2$, the system $(\mathrm{F} 1)(\mathrm{F} 2)$ coincides with the one obtained in [7].

We can easily solve (F2) by noting the above remark (ii).

Proposition 4.2.5. For $\lambda \in P_{\ell}$ and $q \in D^{*}$, the functions 


$$
\Theta(\xi, q)^{\lambda} \chi_{\mu}^{(\ell-2 \lambda)}(\xi, q) \quad\left(\mu=0, \frac{1}{2}, \ldots, \frac{\ell-2 \lambda}{2}\right)
$$

form a basis of the solution space of (F2).

\subsection{Some Solutions}

In this subsection we determine the trace of vertex operators explicitly when $L=\ell-2 \lambda \leq 1$, by solving the differential equations (F1) and (F2).

Case $L=0$ :

In this case the space $\mathfrak{F}_{1}^{r}(\lambda)$ is spanned by the single function

$$
\phi_{\frac{\lambda}{2}}(\xi, q)=\operatorname{Tr}_{\mathscr{H} \frac{\lambda}{2}} \varphi\left(|0\rangle_{\frac{\lambda}{2}} ; 1\right) q^{L_{0}-\frac{c_{\nu}}{24} \xi^{\frac{H}{2}}}
$$

On the other hand, by Proposition 4.2.5, any solution of (F2) is given in the following form:

$$
\phi(\xi, q)=a(q) \Theta(\xi, q)^{\lambda} \chi_{0}^{(0)}(\xi, q)=a(q) \Theta(\xi, q)^{\lambda}
$$

with some function $a(q)$, and the equation (F1) now implies $\partial_{q} a(q)=0$. Therefore, we have

$$
\phi_{\frac{\lambda}{2}}(\xi, q)=\Theta(\xi, q)^{\lambda}
$$

under the appropriate normalization.

Case $L=1$ :

The space $\mathfrak{F}_{1}^{r}(\lambda)$ has dimension 2 and it is spanned by

$$
\phi_{\mu}(\xi, q)=\operatorname{Tr}_{\mathscr{H}_{\mu}} \varphi\left(|0\rangle_{\mu} ; 1\right) q^{L_{0}-\frac{c_{\nu}}{24}} \xi^{\frac{H}{2}} \quad\left(\mu=\frac{\lambda}{2}, \frac{\lambda+1}{2}\right) .
$$

On the other hand, by substuting

$$
a_{0}(q) \Theta(\xi, q)^{\lambda} \chi_{0}^{(1)}(\xi, q)+a_{1}(q) \Theta(\xi, q)^{\lambda} \chi_{\frac{1}{2}}^{(1)}(\xi, q)
$$

for $\phi(\xi, q)$ in (F1), and using (F1) for $L=1, \lambda=0$, we find that the functions

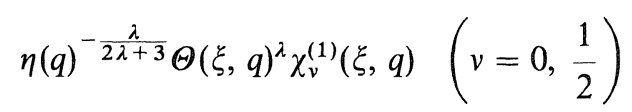

are solutions of the system. By comparing the exponents of $q$, we conclude

$$
\begin{aligned}
& \phi_{\frac{\lambda}{2}}(\xi, q)=\eta(q)^{-\frac{\lambda}{2 \lambda+3}} \Theta(\xi, q)^{\lambda} \chi_{0}^{(1)}(\xi, q), \\
& \phi_{\frac{\lambda+1}{2}}(\xi, q)=\eta(q)^{-\frac{\lambda}{2 \lambda+3}} \Theta(\xi, q)^{\lambda} \chi_{\frac{1}{2}}^{(1)}(\xi, q) \text {. }
\end{aligned}
$$




\section{Acknowledgements}

I would like to thank T. Miwa for stimulating encouragement and inspiring discussions. I am also grateful to M. Kashiwara, H. Ooguri, A. Tsuchiya and K. Ueno for useful suggestions and advice.

\section{References}

[1] Bernard, D., On the Wess-Zumino-Witten models on the torus, Nucl. Phys., B303 (1988), 77-93.

[2] - On the Wess-Zumino-Witten models on Riemann surfaces, Nucl. Phys., B309 (1988), 145-174.

[3] Bernard, D. and Felder, G., Fock representations and BRST cohomology in SL(2) current algebra, Comm. Math. Phys., 127 (1990), 145-168.

[4] Belavin, A. A., Polyakov, A. M. and Zamolodchikov, A. B., Infinite dimensional conformal symmetry in two-dimensional quantum field theory, Nucl. Phys., B241 (1984), 333-380.

[5] Etingof, P. and Kirillov, Jr., A., On the affine analogue of Jack's and Macdonald's polynomials, Yale preprint (1994), hep-th/9403168, to appear Duke Math. J..

[6] Eguchi, T. and Ooguri, H., Conformal and current algebras on a general Riemann surface, Nucl. Phys., B282 (1987), 308-328.

[7] , Differential equations for characters of Virasoro and affine Lie algebras, Nucl. Phys., B313 (1989), 482-508.

[8] Felder, G., Conformal field theory and integrable systems associated to elliptic curves, to appear in th Proceeding of the International Congress of Mathematicians, Zurich (1994).

[9] Felder, G. and Wieczerkowski, C., Conformal blocks on elliptic curves and the KnizhnikZamolodchikov-Bernard equations, hep-th/941104 (1994).

[10] Kac, V., Infinite dimensional Lie algebras, Cambridge University Press., Third edition, 1990.

[11] Knizhnik, V. Z. and Zamolodchikov, A. B., Current algebra and Wess-Zumino models in two dimensions, Nucl. Phys., B247 (1984), 83-103.

[12] Tsuchiya, A. and Kanie, Y., Vertex operators in conformal field theory on $\mathbb{P}^{1}$ and monodromy representations of braid group, Adv. Stud. in Pure Math., 16 (1988), 297.

[13] Tsuchiya, A., Ueno, K. and Yamada, Y., Conformal field theory on universal family of stable curves with gauge symmetries, Adv. Stud. in Pure Math., 19 (1989), 459-566. 
